\title{
FSLLRY-NH2 Improves Neurological Outcome After Cardiac Arrest in Rats
}

\author{
Umut OCAK ${ }^{1,2, *}$, Pinar ESER OCAK ${ }^{2,3}$, Lei HUANG ${ }^{2, * *}$, John H. ZHANG ${ }^{2, *, * * *}$ \\ ${ }^{1}$ University of Health Sciences, Bursa Yuksek Ihtisas Training and Research Hospital, Department of Emergency Medicine, Bursa, Turkey \\ ${ }^{2}$ Loma Linda University School of Medicine, Department of Physiology and Pharmacology, Loma Linda, California, USA \\ ${ }^{3}$ Uludag University, School of Medicine, Department of Neurosurgery, Bursa, Turkey
}

Corresponding author: John H. ZHANG johnzhang3910@yahoo.com

\section{ABSTRACT}

AIM: To evaluate the effect of FSLLRY-NH2, a protease-activated receptor 2 (PAR2) inhibitor, on neurocognitive impairment and hippocampal neuronal degeneration in the setting of asphyxial cardiac arrest (ACA)-induced global cerebral ischemia (GCI) in rats.

MATERIAL and METHODS: A total of 43 Sprague-Dawley male rats were used. Shams and rats resuscitated from 9 minutes of ACA were randomized to two separate experiments including time course and short-term neurological outcomes. FSLLRY-NH2 (50 microgram $[\mu \mathrm{g}]$ per rat) was administered intranasally at 1 hour postresuscitation. Neurological function and hippocampal neuronal degeneration were evaluated after ACA.

RESULTS: Significant neurological function decline and hippocampal neuron degeneration were observed in ACA animals as compared with the shams. Treatment with FSLLRY-NH2 significantly improved neurological outcome and reduced the number of degenerating hippocampal neurons after ACA.

CONCLUSION: Targeting PAR2 may be a novel therapeutic approach in the management of neurological dysfunction after cardiac arrest-associated ischemic injury.

KEYWORDS: Cardiac arrest, cognitive, global cerebral ischemia, PAR2

\section{- INTRODUCTION}

$\longrightarrow$ ardiac arrest (CA) is associated with significant mortality and severe neurofunctional impairment $(3,7)$. In cases of successful resuscitation, return of spontaneous circulation (ROSC) causes ischemia or reperfusion injury in the globally ischemic brain. As a result, global cerebral ischemia $(\mathrm{GCl})$, which occurs during the arrest period and subsequent reperfusion (27), causes irreversible brain damage (17), and results in unfavorable neurological outcomes in CA survivors (14).

Given the susceptibility of particular brain regions to ischemia, such as the cornu ammonis 1 (CA1) region of the hippocampus (16), cognitive dysfunction is an important consequence of CA-induced $\mathrm{GCl}(11,34,37)$. Among many other injury mechanisms, neuroinflammation has long been implicated in neurodegeneration and cognitive disability $(5,23,26)$ as well as $\mathrm{GCl}$-associated neurological dysfunction $(4,21)$. Despite particular interest of a large number of research studies in CA-associated neurological impairment, including cognitive dysfunction, effective strategies are yet to be elucidated.

Protease-activated receptor 2 (PAR2) is a member of the PAR family that is widely expressed in the resident cells of the central nervous system (20). Emerging evidence has pointed out that activation of PAR2 is related to neuroinflammation and neurodegeneration $(12,18,35)$. Moreover, data obtained from both in vivo and in vitro studies underlined augmented expression of PAR2 in the brain after ischemic injury $(25,36)$. Based on this background and given the potential involvement of neuroinflammation in CA-associated cognitive

\footnotetext{
Umut OCAK (1) : 0000-0002-6549-4246 Lei HUANG (1) : 0000-0002-7211-8337

Pinar ESER OCAK (1) : 0000-0003-0132-9927 John H. ZHANG (1) : 0000-0002-4319-4285
} 
Ocak U. et al: Role of FSLLRY-NH2 on Neurological Outcome

decline (4), we hypothesized that inhibition of PAR2 could attenuate neurological impairment and hippocampal neuronal degeneration in the setting of asphyxial CA (ACA)-induced $\mathrm{GCl}$.

\section{MATERIAL and METHODS}

\section{Animals and Rat Models of ACA}

All experimental procedures performed in the current study were approved by the Institutional Animal Care and Use Committee at Loma Linda University, Loma Linda, California, USA. All experiments conducted were in accordance with the National Institutes of Health Guide for the Care and Use of Laboratory Animals. The results were reported according to the ARRIVE guidelines.

A total of 43 adult male Sprague-Dawley rats (450-500 g; Envigo, Indiana, USA) were used for this study. The animals were housed in a humidity- and temperature-controlled facility with 12 hours light and dark cycle and ad libitum food and water access.

The animals were randomly divided into sham $(n=10)$, and ACA groups $(n=33)$. Rats were anesthetized deeply were anesthetized deeply with pentobarbital (intraperitoneal, 45 mg/kg; Virbac AH, Inc., Fort Worth, Texas, USA). First, endotracheal intubation using a 14-gauge plastic catheter under laryngoscopy was performed to the animals. Next, the left femoral artery and vein were exposed through a 1-centimeter skin incision along the left groin followed by blunt dissection of the surrounding connective tissue. Then, a polyethylene (PE) 50 catheter (Becton Dickinson, Franklin Lakes, New Jersey, USA) was inserted in the femoral artery and connected to a pressure transducer. Another PE 50 catheter was inserted in the femoral vein for drug administration. A rectal probe (Model BAT-12, Physitemp Instrument Inc, Clifton, New Jersey, USA) was inserted to monitor rectal body temperature during the procedure. Electrocardiogram (Lead II) was recorded continuously. The rats were mechanically ventilated (respiratory frequency, 100 beats per minute; tidal volume, $\left.0.55 \mathrm{~mL} / 100 \mathrm{~g} ; \mathrm{FiO}_{2}, 21 \%\right)$ for 15 minutes before the induction of ACA. ACA was induced by chemical neuromuscular blockade with intravenous vecuronium (2 mg/kg; Mylan Institutional LLC., Rockford, Illinois, USA). The ventilator was disconnected, and the intubation tube was obstructed. After 9 minutes of untreated asphyxia, resuscitation was initiated by unclamping the tracheal tube, administering epinephrine $(7.5 \mu \mathrm{g} / \mathrm{kg}$; International Medication System, LLC., South El Monte, California, USA) and sodium bicarbonate (1 mEq/kg; Hospira, Lake Forest, Illinois, USA), and applying precordial compressions with a pneumatically driven mechanical chest compressor as well as mechanical ventilation with $100 \%$ oxygen at a ratio of 2:1. Mean arterial pressure of $>60 \mathrm{mmHg}$ and return of sinus rhythm for 5 minutes were defined as successful resuscitation and ROSC. The animals were excluded from the study if ROSC was achieved after 5 minutes of resuscitation or if ROSC was not achieved at all. After ROSC, mechanical ventilation was continued for 30 minutes with $100 \%$ oxygen and was gradually reduced to $21 \%$ every 10 minutes within 1 hour. The animals were weaned from the ventilator; endotracheal tube and all catheters were removed 1 hour after resuscitation. The wound was sutured, and the animals were allowed to recover. Their body temperature was maintained at $36.5^{\circ} \mathrm{C} \pm 0.5{ }^{\circ} \mathrm{C}$ using a heating lamp. The sham group underwent same surgical procedures and baseline ventilation without inducing ACA. Electrocardiogram, end-tidal carbon dioxide, and arterial blood pressure were continuously recorded during the whole procedure from 15 minutes before asphyxia induction until 1 hour after ROSC on a PC-based data-acquisition system supported by WINDAQ software (DATAQ, Akron, Ohio, USA).

\section{Experimental Design}

Two main experiments including time course or cellular colocalization of PAR2 and short-term (7 day) outcome of PAR2 inhibitor treatment were performed. The number and distribution of animals per experimental group are presented in Table I.

Experiment 1 (Time course study and cellular colocalization of PAR2)

The temporal expression of endogenous brain PAR2 was evaluated through Western blot at $6,12,24$, and 72 hours after ACA ( $n=4$ per group). Cellular colocalization of PAR2 with microglia marker calcium-binding adaptor molecule 1 (Iba1), astrocyte marker glial fibrillary acidic protein (GFAP), or neuron marker (NeuN-neuronal nuclei) was evaluated through double-immunofluorescence staining in sham and ACA animals at 24 hours after the injury ( $n=1$ per group).

\section{Experiment 2 (Short-term outcome)}

The treatment effects of PAR2 inhibition on ACA outcomes were examined. Selective PAR2 inhibitor (FSLLRY-NH2; 50 $\mu \mathrm{g} / \mathrm{rat}$; Tocris Bioscience, Minneapolis, Minnesota, USA) was intranasally administered at 1 hour postresuscitation. Short-term neurological function was assessed through neurological deficit score (NDS) (24-hour, 48-hour, and 72hour postresuscitation), and neurocognition was evaluated using T-maze test (7 days) after ACA. The effect of FSLLRY$\mathrm{NH} 2$ on hippocampal neuron degeneration was evaluated through Fluoro-Jade C (FJC) staining 7 days after ACA.

\section{Intranasal Drug Administration}

One hour after ACA, FSLLRY-NH2 was diluted in $20 \%$ ethanol and administered intranasally as previously described (24). Briefly, the animals were placed in supine position under $2 \%$ isoflurane anesthesia. A total volume of 30 microliters $(\mu \mathrm{L})$ of vehicle $(20 \%$ ethanol) or FSLLRY-NH2 $(50 \mu \mathrm{g} / \mathrm{rat})$ in $20 \%$ ethanol was administered to the left and right nares, alternately administering $5 \mu \mathrm{L}$ in one naris every 2 minutes for a period of 10 minutes.

\section{Assessment of Neurological Function}

Neurological functions were assessed by a blinded investigator. Consciousness, respiration, corneal reflex, auditory reflex, motor function, and behavior were evaluated through NDS at 24,48 , and 72 hours after ACA. Higher scores indicated worse performance. The total test score ranged between 0 (normal) 
Table I: The Number and Distribution of the Animals Included for the Experiments

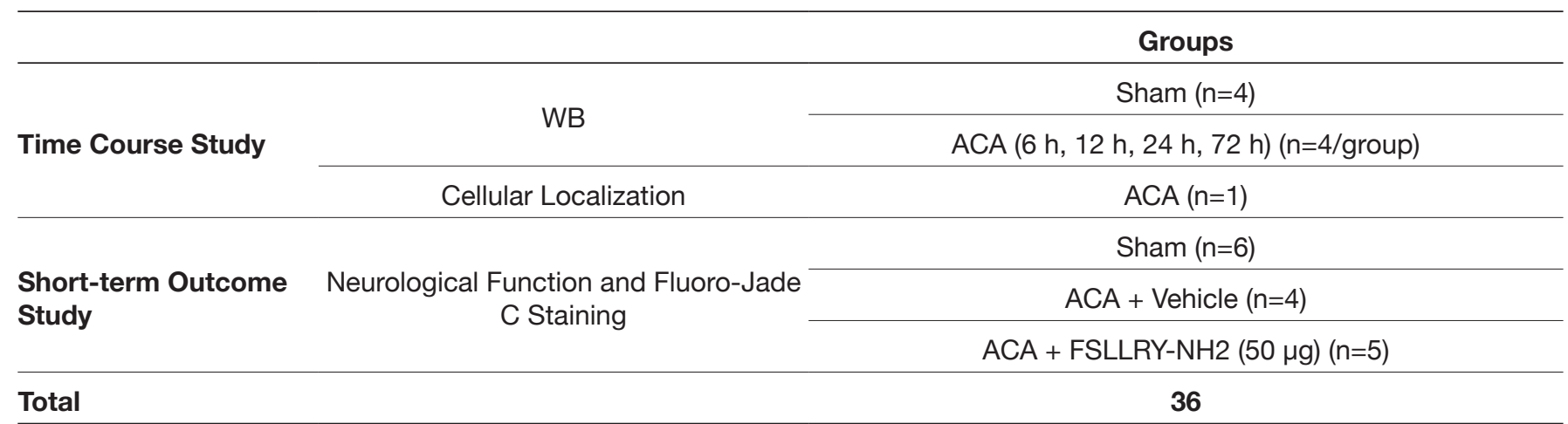

ACA: Asphyxial cardiac arrest, h: Hours, IHC: Immunohistochemistry, ROSC: Return of spontaneous circulation, WB: Western blot.

and 500 (coma) (10). Cognitive deficits were evaluated using T-maze spontaneous alternation test 7 days after ACA. For this test, the percentage of spontaneous alternation (number of turns in each goal arm) was recorded, and the results were expressed as percent with respect to $50 \%$ reference $(15,22)$.

\section{Histological Analysis}

Briefly, the rats underwent transcardiac perfusion with $100 \mathrm{~mL}$ of chilled phosphate-buffered saline (PBS; $0.01 \mathrm{M}$; pH 7.4), followed by $100 \mathrm{~mL}$ of $10 \%$ formalin under deep anesthesia. The brains were quickly removed, fixed in $10 \%$ formalin at $4{ }^{\circ} \mathrm{C}$ for 24 hours, and then dehydrated in 30\% sucrose for another 72 hours. After being embedded into OCT (Scigen Scientific Gardena, California, USA), the 10 micrometer coronal brain sections were obtained at 3.8 millimeter $(\mathrm{mm})$ posterior to the bregma on a cryostat (CM3050S; Leica Microsystems, Bannockburn, III, Germany).

Double-immunofluorescence staining was performed as previously described (45). Briefly, the slices were washed in $0.01 \mathrm{M}$ of PBS ( $3 \times 5$ minutes) and blocked with $5 \%$ donkey serum at room temperature for 1 hour. Then, the slices were incubated overnight at $4{ }^{\circ} \mathrm{C}$ with the following primary antibodies: anti-PAR2 (1:200), anti-lba1 (1:200), anti-GFAP (1:200), and anti-NeuN (1:200) (all from Abcam, Cambridge, Massachusetts, USA). The sections were incubated with appropriate fluorescence-conjugated secondary antibodies (1:100, Jackson Immuno Research, West Grove, Pennsylvania, USA) at room temperature for 1 hour the following day. The slides were then visualized and photographed under a fluorescence microscope (BZ-X800; Keyence Corporation, Itasca, Illinois, USA).

As previously described (24, 30), FJC Ready-to-Dilute Staining Kit (Biosensis, USA) was used for FJC staining based on the manufacturer's instructions. ImageJ software (ImageJ 1.5, NIH, USA) was used to count the number of FJC-positive neurons. Data gathered were presented as the number of FJC-positive neurons expressed in per $\mathrm{mm}^{2}$ in the fields.

\section{Western Blot Analysis}

Western blot analysis was performed as previously described $(38,42,44)$. Briefly, the animals underwent transcardiac perfusion of $100 \mathrm{~mL}$ of ice-cold PBS (0.01 M; pH 7.4) under deep sedation before the brains were quickly removed. Brain samples were snap frozen in liquid nitrogen and stored at a temperature of $-80^{\circ} \mathrm{C}$ until use. During sample preparation, tissues were homogenized in RIPA Lysis Buffer (Santa Cruz Biotechnology Inc., Texas, USA) and centrifuged at 14,000 $\mathrm{g}$ at $4^{\circ} \mathrm{C}$ for 30 minutes. Using detergent-compatible assay (DC protein assay; Bio-Rad Laboratories, California, USA), protein concentrations of the supernatants were measured. Then, equal amounts of protein $(30 \mu \mathrm{g})$ were separated through SDS-PAGE gel electrophoresis and transferred to nitrocellulose membranes. Membranes were blocked with $5 \%$ nonfat-blocking grade milk (Bio-Rad, Hercules, California, USA) and incubated overnight at $4^{\circ} \mathrm{C}$ with the following primary antibodies: anti-PAR2 (1:500) and anti- $\beta$ actin (1:2000) (all from Santa Cruz Biotechnology, Dallas, Texas, USA). The membranes were incubated with the appropriate secondary antibody (1:2000, Santa Cruz, Dallas, Texas, USA) at room temperature for 2 hours the following day. The bands were visualized with ECL Plus chemiluminescence reagent kit (Amersham Bioscience, Pennsylvania, USA). ImageJ software (Image J 1.4, NIH, USA) was used to quantify the densities of the immunoblots. $\beta$-actin was used as internal control.

\section{Statistical Analysis}

All data were presented as mean \pm standard deviation (mean \pm SD) and were analyzed using GraphPad Prism 7 (GraphPad Software, San Diego, California, USA). One-way ANOVA followed by Tukey's post hoc test was used for comparison among multiple groups. A p value $<0.05$ was considered statistically significant.

\section{RESULTS}

\section{Mortality}

No mortality was observed in the sham group. In the ACA group $(n=33)$, ROSC was achieved in 26 rats $(78.8 \%)$. Only the resuscitated rats in the ACA group $(n=26)$ were further included for our experiments. Post-ROSC mortality was $0 \%$ $(n=0)$. Moreover, no significant difference in the mortality rates among the ACA groups was observed $(p=0.7075)$. 


\section{PAR2 Expression Significantly Increased in the Brain After ACA}

Time course of PAR2 expression was evaluated in sham and ACA animals using Western blot, which revealed that PAR2 levels were significantly increased in the brain after ACA. The increase in protein levels of PAR2 started as early as 6 hours, and the elevation sustained until 72 hours after ACA as compared with sham-operated rats (Figure 1A, B).

Double-immunofluorescence staining of PAR2 for its cellular colocalization revealed that PAR2 was expressed in the microglia, astrocytes, and neurons 24 hours after ACA (Figure 2A-C).

\section{Inhibition of PAR2 with FSLLRY-NH2 Attenuated Neurological Impairment for 3 Days After ACA}

Significant deterioration in neurological function was observed in ACA animals as compared with the shams, which was defined by the higher NDS at 24, 48, and 72 hours after ACA. FSLLRY-NH2 treatment at a dose of $50 \mu \mathrm{g}$ effectively improved neurological impairment with significantly lower NDS at all time points after ACA as compared with vehicletreated ACA rats. (Figure $3 A-C$ ).

\section{Inhibition of PAR2 with FSLLRY-NH2 Reduced Hippocampal Neuronal Death And Improved Neurocognitive Function 7 Days After ACA}

FJC staining revealed that ACA was associated with marked increase in neuronal degeneration within the hippocampal CA1 region 7 days after ACA. The inhibition of PAR2 with FSLLRY$\mathrm{NH} 2$ at a dose of $50 \mu \mathrm{g}$ significantly reduced the number of FJC-positive degenerating neurons within the hippocampal CA1 region as compared with vehicle + ACA group (Figure 4A, $B)$. Spontaneous alternation as defined by the T-maze test was significantly lower in ACA + vehicle group as compared with shams, suggesting neurocognitive dysfunction. Consistently, a $50 \mu \mathrm{g}$ treatment with FSLLRY-NH2 significantly improved the performance in T-maze test as compared with vehicletreated ACA animals (Figure 4C).

\section{DISCUSSION}

In this study, we focused on the potential neuroprotective effect of PAR2 inhibition using an ACA rat model. Our findings were as follows: 1) PAR2 is expressed in the brain both in rats subjected to sham operation and ACA; 2) PAR2 expression significantly increased in the brain after ACA-induced $\mathrm{GCl}$; 3) ACA resulted in significantly increased neuron degeneration in the hippocampal CA1 region and short-term neuronal dysfunction; 4) Inhibition of PAR2 with its selective inhibitor (FSLLRY-NH2) significantly reduced hippocampal neuron degeneration and improved short-term neurocognitive decline caused by ACA.

As outlined in the introduction section, neurocognitive impairment is a well-known consequence of CA. This is due to the vulnerability of the hippocampus, particularly the pyramidal neurons in the hippocampal CA1 region to transient ischemia such as CA-induced $\mathrm{GCl}(8,13,39)$. Consequently, substantial loss of pyramidal neurons in the hippocampal CA1 region but not in the $\mathrm{CA} 2$ or $\mathrm{CA} 3$ region and dentate gyrus was demonstrated after $\mathrm{GCl}(13,39)$. Consistent with the well-known role of the hippocampus in memory formation, cognition, and spatial learning $(2,6,24)$, earlier clinical studies showed that cognitive decline may last up to 3 years after $\mathrm{CA}$-induced $\mathrm{GCl}$ and obviously decreases the patients' quality of life (34). Therefore, strategies targeting improvement of neurological dysfunction after CA are mandatory.

PAR2 belongs to the PAR family, which is a G-protein-coupled receptor family that is activated by cleavage of a section of the amino terminus by serine proteinases (33). PAR2 is widely expressed in the brain, and it is preferentially activated by trypsin and mast cell tryptase, whereas PAR1, PAR3, and PAR4 are preferentially activated by thrombin (31). The activation of PAR2 has been implicated in neuroinflammation and neurodegeneration $(4,9,12)$. The activation of microglial and astrocytic PAR2 was demonstrated to result in the release of proinflammatory cytokines IL-6 and TNF-a in earlier in vitro studies $(40,41)$. In addition, induced PAR2 expression

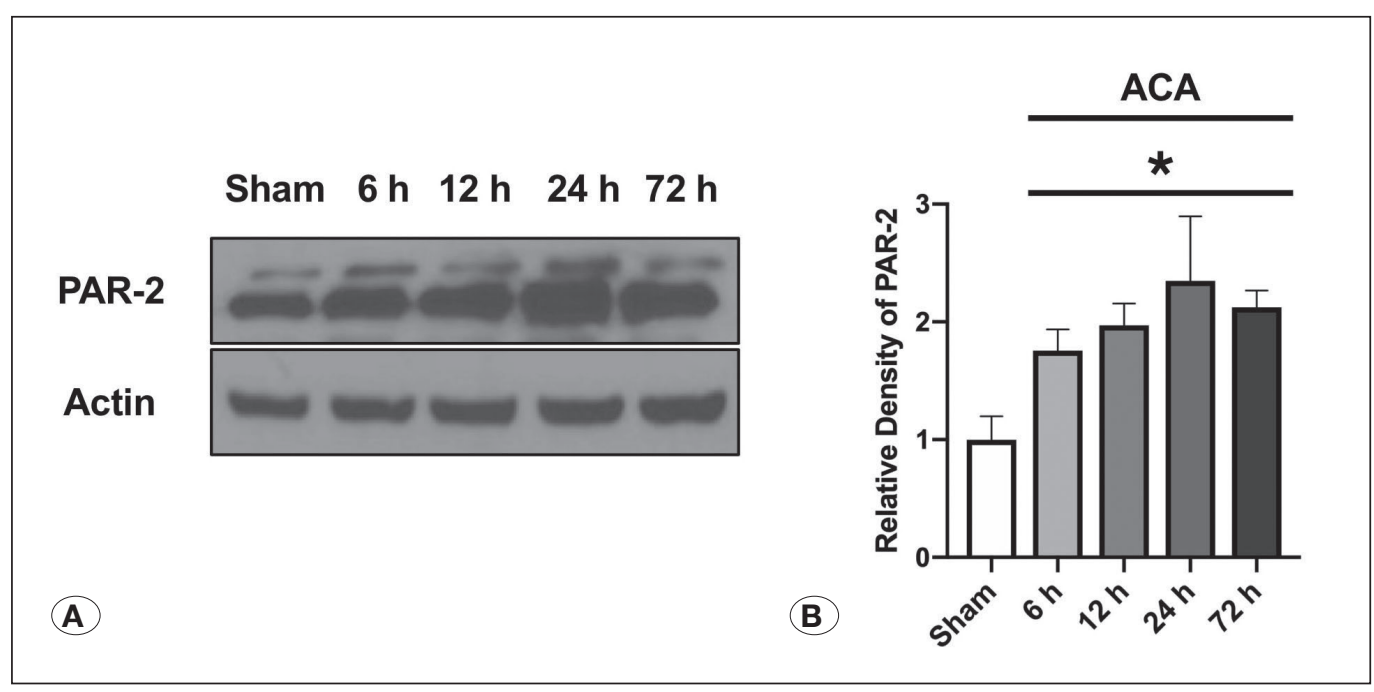

Figure 1: Temporal expression of brain PAR2 following ACA.

Representative western blot images (A), and quantitative analyses of endogenous brain PAR2 (B) over 72 hours following ACA.

A significant increase in protein level of PAR2, starting early after the injury and persisting until 72 hours was observed in animals subjected to ACA compared to the Sham group. (Data are expressed as mean \pm SD. $n=4$ /group. ${ }^{*} p<0.05$ compared to Sham; ANOVA, Tukey). 


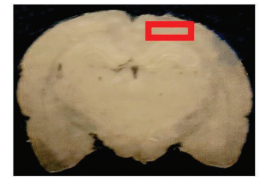

(A)
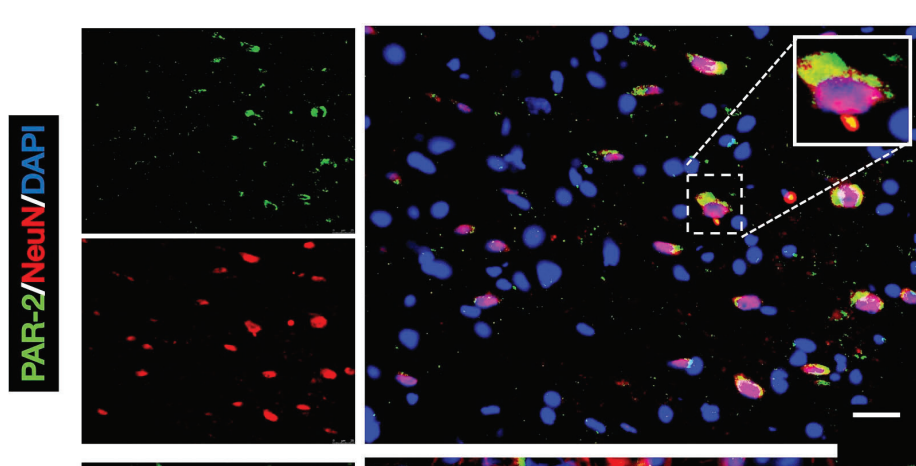

(B)
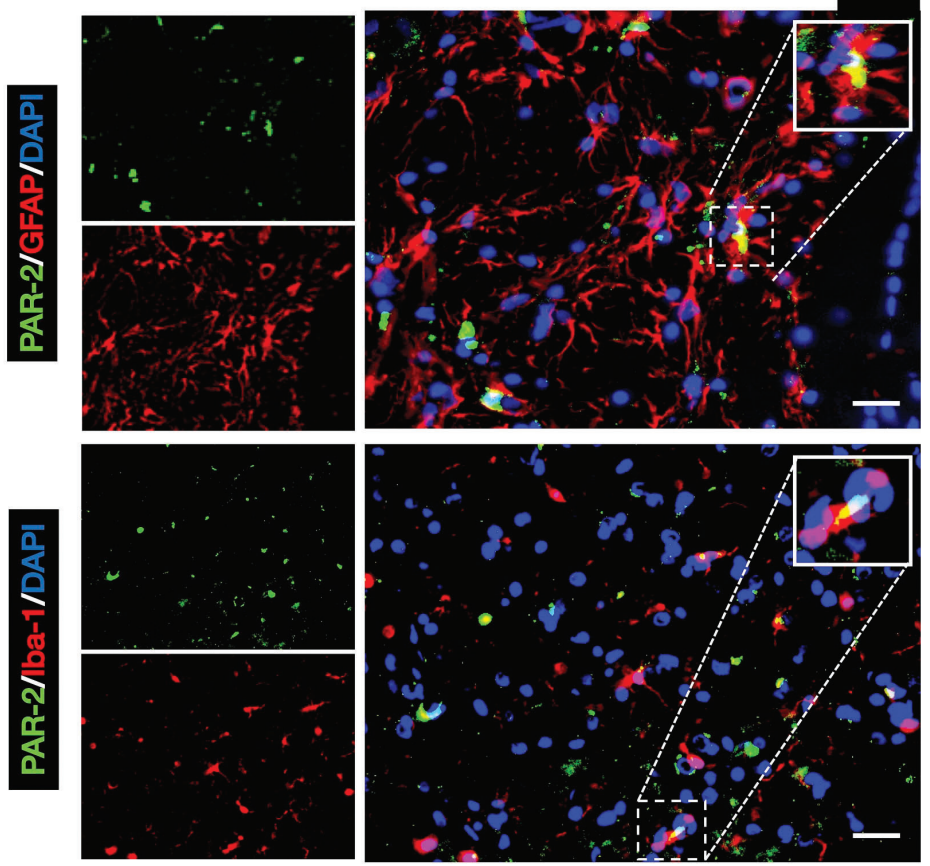

Figure 2: Cellular colocalization of PAR2 in neurons, astrocytes and microglia.

Representative doubleimmunofluorescence staining microphotography at 24 hours following ACA showed that PAR2 (green) co-localized with neuronal, astrocytic and microglial markers NeuN (A), astrocytes (GFAP) (B), and microglia (lba-1) (C) (all red). Nuclei of the cells are stained with DAPI $(\mathbf{A}, \mathbf{B}, \mathbf{C})$ (blue). The location of staining was indicated as small red box in coronal brain slice. (Scale bar $=50 \mu \mathrm{m}, \mathrm{n}=1$ /group).

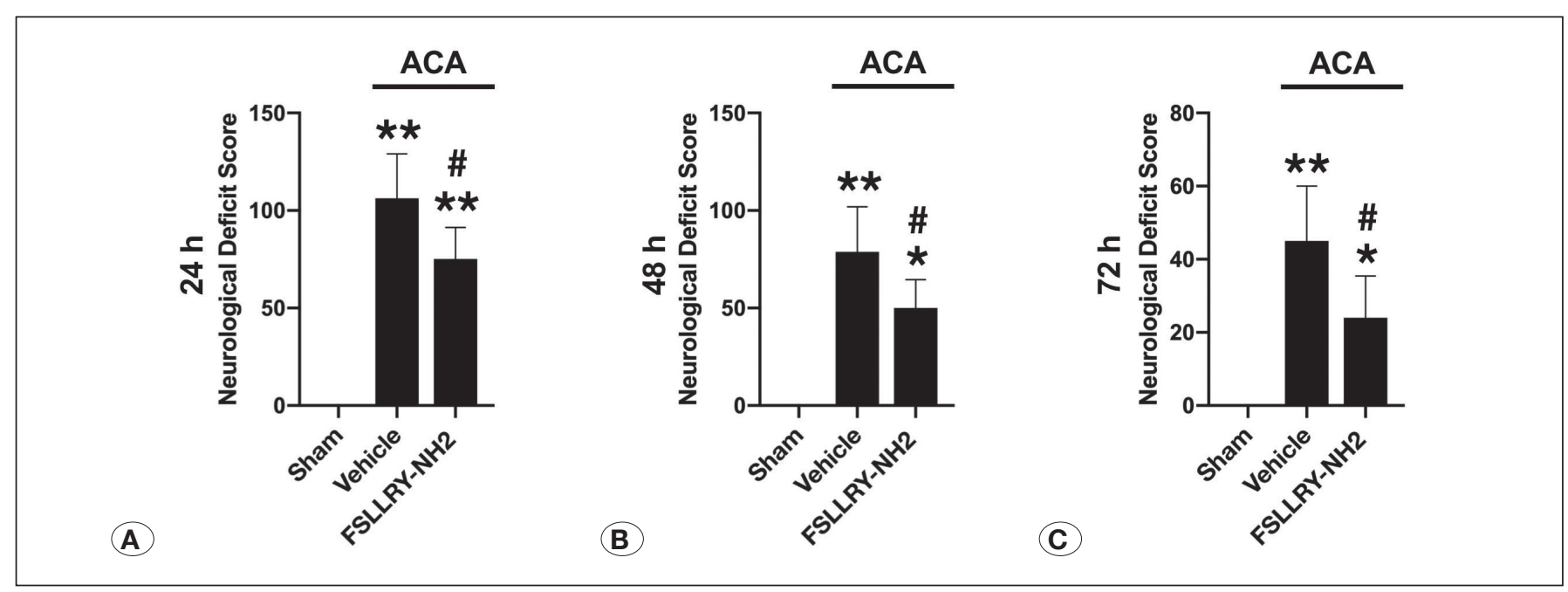

Figure 3: Inhibition of PAR2 with FSLLRY-NH2 improved short-term neurological deficits after ACA. Effect of FSLLRY-NH2 on neurological deficit score (NDS) was assessed at 24 (A), 48 (B) and 72 (C) hours after ACA. ACA was associated with significantly worse neurologic functions compared to the shams at all time points. Inhibition of PAR2 with FSLLRY-NH2 at a dose of $50 \mu \mathrm{g}$ improved NDS at 24,48 and 72 hours after ACA compared to the vehicle treated ACA rats. (Data are expressed as mean \pm SD. $n=4-6 /$ group. ${ }^{* *} p<0.001$ compared to Sham, ${ }^{*} p<0.05$ compared to Sham, \#p<0.05 compared to ACA + vehicle; ANOVA, Tukey). 


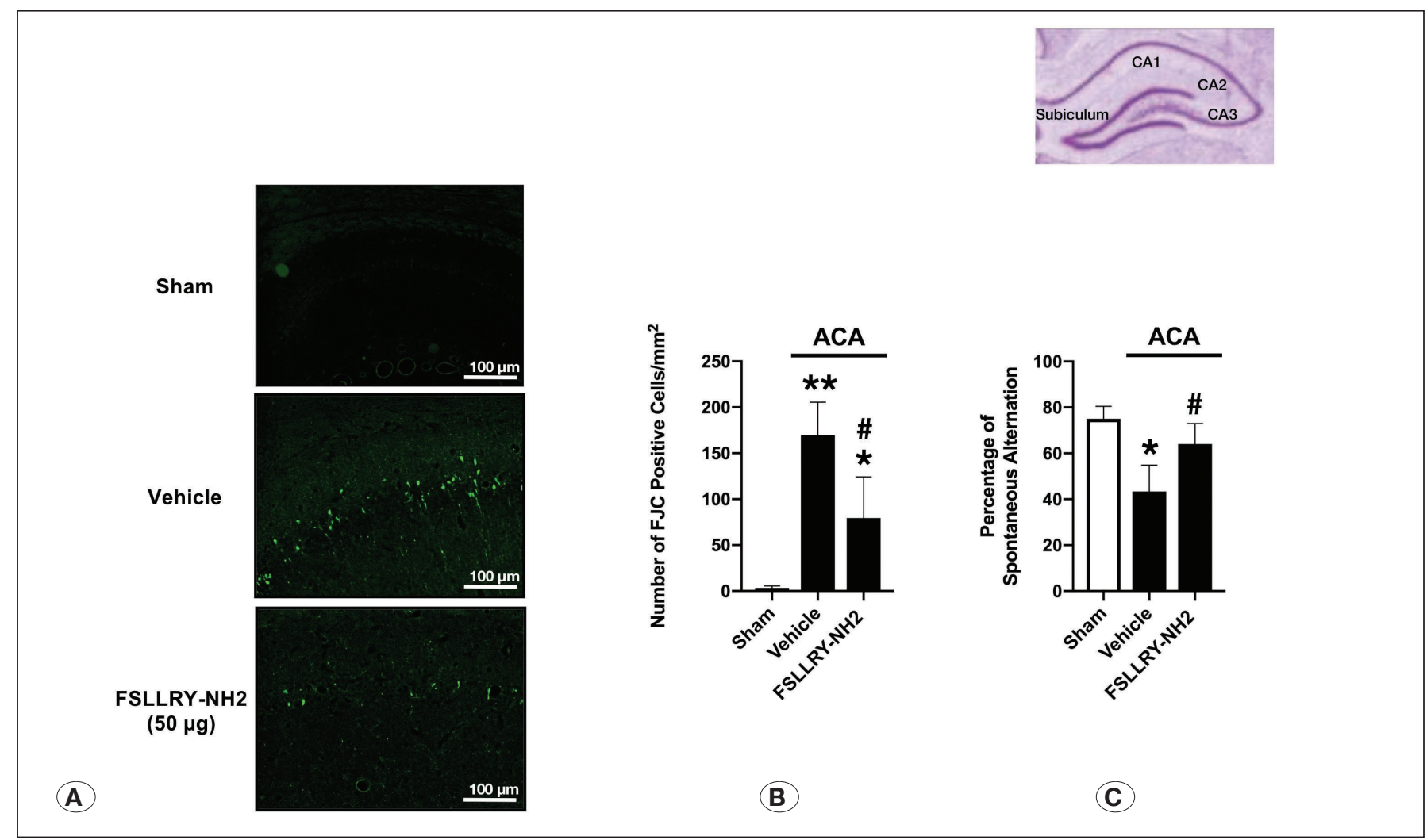

Figure 4: Inhibition of PAR2 with FSLLRY-NH2 reduced the number of FJC-positive degenerating neurons within hippocampus and improved cognitive functions at 7 days after ACA.

Representative FJC staining microphotographs (A), quantitative analyses of FJC-positive cells in (B) hippocampal CA1 region and spontaneous alternation of T-maze test (C). ACA caused significant neuronal degeneration in the CA1 region and a significant decrease in spontaneous alternation of T-maze test at 7 days following ACA. FSLLRY-NH2 at a dose of $50 \mu g$ significantly reduced the number of FJC-positive cells in CA1 region and improved T-maze test performance compared to the vehicle treated ACA rats. (Scale bar=100 $\mu \mathrm{m})$. (Data are expressed as mean $\pm \mathrm{SD}$. $\mathrm{n}=4-6$ /group. ${ }^{\star *} \mathrm{p}<0.001$ compared to Sham, ${ }^{*} \mathrm{p}<0.05$ compared to Sham, \#p<0.05 compared to ACA + vehicle, ANOVA, Tukey).

in response to alpha-synuclein, which is known to trigger neurodegeneration, was reported in the brains of patients with Parkinson's disease (12). Similarly, enhanced expression of PAR2 was found in the brain in patients with Alzheimer's disease (1) as well as multiple sclerosis (28).

Although data regarding the alterations in PAR2 expression after brain ischemia remain controversial, several previous studies demonstrated increased protein levels of PAR2 in severely ischemic brain $(25,36)$. Moreover, PAR2 was shown to be highly expressed in the hippocampus, and its activation negatively affected learning functions and cognition $(19,35)$. Consistent with previous results, we showed in this study that PAR2 is expressed in the resident cells of the brain including microglia, astrocytes, and neurons in the animals subjected to asphyxia-induced CA. We also showed that the expression of PAR2 significantly increased in the brain after CA by using Western blot analysis. In addition, increased neuron degeneration that was observed in the hippocampal CA1 slices obtained from ACA animals 7 days after the injury was effectively reduced by administering a selective inhibitor, FSLLRY-NH2, of PAR2 in our study. Consistently, the animals treated with FSLLRY-NH2 exhibited better performance in neurological tests as defined by lower NDS and also improved neurocognitive performance in T-maze test in the current study.

This study has several limitations. First, the contribution of PAR2 to neuronal apoptosis (32) as well as blood-brain damage (43) was reported in earlier studies, in addition to its proinflammatory effects (29). We did not investigate the detailed mechanisms underlying the neuroprotection provided by PAR2 inhibitor in the setting of GCI after ACA. Second, we have not evaluated long-term effect of PAR2 inhibition against neurological impairments after ACA. Therefore, our future studies will further address these issues.

\section{CONCLUSION}

In summary, inhibition of PAR2 with FSLLRY-NH2 improved short-term neurological impairments and attenuated neuronal degeneration in the hippocampal CA1 region in an ACA rat model. Our findings suggest that targeting brain PAR2 may be a novel therapeutic strategy in managing neurocognitive decline in CA survivors. 


\section{ACKNOWLEDGEMENTS}

This work was funded by LLU School of Medicine-Department of Neurosurgery funding (IACUC Record Number: 8180029).

Preparation for publication of this article is partly supported by Turkish Neurosurgical Society.

The affiliations of authors:

*Bursa City Hospital, Department of Emergency Medicine, Bursa, Turkey

**Loma Linda University School of Medicine, Department of Neurosurgery, Loma Linda, California, USA

***Loma Linda University School of Medicine, Department of Anesthesiology and Neurology, Loma Linda, California, USA

\section{REFERENCES}

1. Afkhami-Goli A, Noorbakhsh F, Keller AJ, Vergnolle N, Westaway D, Jhamandas JH, Andrade-Gordon P, Hollenberg $\mathrm{MD}$, Arab $\mathrm{H}$, Dyck $\mathrm{RH}$, Power C: Proteinase-activated receptor-2 exerts protective and pathogenic cell type-specific effects in Alzheimer's disease. J Immunol 179:5493-5503, 2007

2. Bendel O, Bueters T, von Euler M, Ove Ogren S, Sandin J, von Euler G: Reappearance of hippocampal CA1 neurons after ischemia is associated with recovery of learning and memory. J Cereb Blood Flow Metab 25:1586-1595, 2005

3. Benjamin EJ, Virani SS, Callaway CW, Chamberlain AM, Chang AR, Cheng S, Chiuve SE, Cushman M, Delling FN, Deo R, de Ferranti SD, Ferguson JF, Fornage M, Gillespie C, Isasi CR, Jimenez MC, Jordan LC, Judd SE, Lackland D, Lichtman $\mathrm{JH}$, Lisabeth L, et al: Heart disease and stroke statistics-2018 update: A report from the american heart association. Circulation 137:e67-e492, 2018

4. Chu K, Yin B, Wang J, Peng G, Liang H, Xu Z, Du Y, Fang $\mathrm{M}$, Xia $\mathrm{Q}$, Luo $\mathrm{B}$ : Inhibition of $\mathrm{P} 2 \mathrm{X} 7$ receptor ameliorates transient global cerebral ischemia/reperfusion injury via modulating inflammatory responses in the rat hippocampus. J Neuroinflammation 9:69, 2012

5. Cuenca L, Gil-Martinez AL, Cano-Fernandez L, SanchezRodrigo C, Estrada C, Fernandez-Villalba E, Herrero MT: Parkinson's disease: A short story of 200 years. Histol Histopathol 34:573-591, 2019

6. Delattre C, Bournonville C, Auger F, Lopes R, Delmaire C, Henon $\mathrm{H}$, Mendyk AM, Bombois S, Devedjian JC, Leys D, Cordonnier C, Bordet R, Bastide M: Hippocampal deformations and entorhinal cortex atrophy as an anatomical signature of long-term cognitive impairment: From the MCAO rat model to the stroke patient. Transl Stroke Res 9:294-305, 2018

7. Field JM, Hazinski MF, Sayre MR, Chameides L, Schexnayder SM, Hemphill R, Samson RA, Kattwinkel J, Berg RA, Bhanji F, Cave DM, Jauch EC, Kudenchuk PJ, Neumar RW, Peberdy MA, Perlman JM, Sinz E, Travers AH, Berg MD, Billi JE, Eigel $B$, et al: Part 1: Executive summary: 2010 American Heart Association Guidelines for cardiopulmonary resuscitation and emergency cardiovascular care. Circulation 122:S640-656, 2010
8. Globus MY, Busto R, Martinez E, Valdes I, Dietrich WD, Ginsberg MD: Comparative effect of transient global ischemia on extracellular levels of glutamate, glycine, and gammaaminobutyric acid in vulnerable and nonvulnerable brain regions in the rat. J Neurochem 57:470-478, 1991

9. Hanusova Z, Mosko T, Matej R, Holada K: Precision in the design of an experimental study deflects the significance of proteinase-activated receptor 2 expression in scrapieinoculated mice. J Gen Virol 98:1563-1569, 2017

10. Hendrickx HH, Rao GR, Safar P, Gisvold SE: Asphyxia, cardiac arrest and resuscitation in rats. I. Short term recovery. Resuscitation 12:97-116, 1984

11. Huang L, Applegate li RL, Applegate PM, Gong L, Ocak $\mathrm{U}$, Boling $\mathrm{W}$, Zhang $\mathrm{JH}$ : Inhalation of high-concentration hydrogen gas attenuates cognitive deficits in a rat model of asphyxia induced-cardiac arrest. Med Gas Res 9:122-126, 2019

12. Kempuraj D, Selvakumar GP, Thangavel R, Ahmed ME, Zaheer S, Kumar KK, Yelam A, Kaur H, Dubova I, Raikwar SP, Iyer SS, Zaheer A: Glia maturation factor and mast cell-dependent expression of inflammatory mediators and proteinase activated receptor-2 in neuroinflammation. $\mathrm{J}$ Alzheimers Dis 66:1117-1129, 2018

13. Kirino T, Sano K: Selective vulnerability in the gerbil hippocampus following transient ischemia. Acta Neuropathol 62:201-208, 1984

14. Laver S, Farrow C, Turner D, Nolan J: Mode of death after admission to an intensive care unit following cardiac arrest. Intensive Care Med 30:2126-2128, 2004

15. Li P, Zhao G, Ding Y, Wang T, Flores J, Ocak U, Wu P, Zhang T, Mo J, Zhang JH, Tang J: Rh-IFN-alpha attenuates neuroinflammation and improves neurological function by inhibiting NF-kappaB through JAK1-STAT1/TRAF3 pathway in an experimental GMH rat model. Brain Behav Immun 79:174185,2019

16. Lim C, Alexander MP, LaFleche G, Schnyer DM, Verfaellie M: The neurological and cognitive sequelae of cardiac arrest. Neurology 63:1774-1778, 2004

17. Lin CS, Polsky K, Nadler JV, Crain BJ: Selective neocortical and thalamic cell death in the gerbil after transient ischemia. Neuroscience 35:289-299, 1990

18. Liu P, Sun L, Zhao XL, Zhang P, Zhao XM, Zhang J: PAR2mediated epigenetic upregulation of alpha-synuclein contributes to the pathogenesis of Parkinsons disease. Brain Res 1565:82-89, 2014

19. Lohman RJ, Jones NC, O’Brien TJ, Cocks TM: A regulatory role for protease-activated receptor-2 in motivational learning in rats. Neurobiol Learn Mem 92:301-309, 2009

20. Luo W, Wang Y, Reiser G: Protease-activated receptors in the brain: Receptor expression, activation, and functions in neurodegeneration and neuroprotection. Brain Res Rev 56:331-345, 2007

21. Ma Y, Chen C, Zhang S, Wang Q, Chen H, Dong Y, Zhang Z, Li Y, Niu Z, Zhu T, Yu H, Liu B: RNase alleviates neurological dysfunction in mice undergoing cardiac arrest and cardiopulmonary resuscitation. Oncotarget 8:53084-53099, 2017 
Ocak U. et al: Role of FSLLRY-NH2 on Neurological Outcome

22. Matchett GA, Calinisan JB, Matchett GC, Martin RD, Zhang $\mathrm{JH}$ : The effect of granulocyte-colony stimulating factor in global cerebral ischemia in rats. Brain Res 1136:200-207, 2007

23. McManus RM, Heneka MT: Role of neuroinflammation in neurodegeneration: New insights. Alzheimers Res Ther 9:14, 2017

24. Mo J, Enkhjargal B, Travis ZD, Zhou K, Wu P, Zhang G, Zhu Q, Zhang T, Peng J, Xu W, Ocak U, Chen Y, Tang J, Zhang J, Zhang JH: AVE 0991 attenuates oxidative stress and neuronal apoptosis via Mas/PKA/CREB/UCP-2 pathway after subarachnoid hemorrhage in rats. Redox Biol 20:75-86, 2019

25. Morihara R, Yamashita T, Kono S, Shang J, Nakano Y, Sato K, Hishikawa N, Ohta Y, Heitmeier S, Perzborn E, Abe K: Reduction of intracerebral hemorrhage by rivaroxaban after tPA thrombolysis is associated with downregulation of PAR-1 and PAR-2. J Neurosci Res 95:1818-1828, 2017

26. Musella A, Gentile A, Rizzo FR, De Vito F, Fresegna D, Bullitta S, Vanni V, Guadalupi L, Stampanoni Bassi M, Buttari F, Centonze D, Mandolesi G: Interplay between age and neuroinflammation in multiple sclerosis: Effects on motor and cognitive functions. Front Aging Neurosci 10:238, 2018

27. Nolan JP, Neumar RW, Adrie C, Aibiki M, Berg RA, Bottiger BW, Callaway C, Clark RS, Geocadin RG, Jauch EC, Kern KB, Laurent I, Longstreth WT, Merchant RM, Morley P, Morrison LJ, Nadkarni V, Peberdy MA, Rivers EP, Rodriguez-Nunez A, Sellke FW, et al: Post-cardiac arrest syndrome: epidemiology, pathophysiology, treatment, and prognostication. A Scientific Statement from the International Liaison Committee on Resuscitation; the American Heart Association Emergency Cardiovascular Care Committee; the Council on Cardiovascular Surgery and Anesthesia; the Council on Cardiopulmonary, Perioperative, and Critical Care; the Council on Clinical Cardiology; the Council on Stroke. Resuscitation 79:350-379, 2008

28. Noorbakhsh F, Tsutsui S, Vergnolle N, Boven LA, Shariat N, Vodjgani M, Warren KG, Andrade-Gordon P, Hollenberg MD, Power C: Proteinase-activated receptor 2 modulates neuroinflammation in experimental autoimmune encephalomyelitis and multiple sclerosis. J Exp Med 203:425435, 2006

29. Ocak U, Ocak PE, Wang A, Zhang JH, Boling W, Wu P, Mo J, Zhang T, Huang L: Targeting mast cell as a neuroprotective strategy. Brain Inj 33:723-733, 2019

30. Okada T, Enkhjargal B, Travis ZD, Ocak U, Tang J, Suzuki H, Zhang JH: FGF-2 Attenuates Neuronal Apoptosis via FGFR3/ PI3K/Akt Signaling Pathway After Subarachnoid Hemorrhage. Mol Neurobiol 56(12):8203-8219, 2019

31. Ossovskaya VS, Bunnett NW: Protease-activated receptors: Contribution to physiology and disease. Physiol Rev 84:579621,2004

32. Park GH, Jeon SJ, Ko HM, Ryu JR, Lee JM, Kim HY, Han SH, Kang YS, Park SH, Shin CY, Ko KH: Activation of microglial cells via protease-activated receptor 2 mediates neuronal cell death in cultured rat primary neuron. Nitric Oxide 22:18-29, 2010

33. Ramachandran R, Noorbakhsh F, Defea K, Hollenberg MD: Targeting proteinase-activated receptors: Therapeutic potential and challenges. Nat Rev Drug Discov 11:69-86, 2012
34. Roine RO, Kajaste S, Kaste M: Neuropsychological sequelae of cardiac arrest. JAMA 269:237-242, 1993

35. Smith-Swintosky VL, Cheo-Isaacs CT, D'Andrea MR, Santulli RJ, Darrow AL, Andrade-Gordon P: Protease-activated receptor-2 (PAR-2) is present in the rat hippocampus and is associated with neurodegeneration. J Neurochem 69:18901896, 1997

36. Striggow F, Riek-Burchardt M, Kiesel A, Schmidt W, HenrichNoack P, Breder J, Krug M, Reymann KG, Reiser G: Four different types of protease-activated receptors are widely expressed in the brain and are up-regulated in hippocampus by severe ischemia. Eur J Neurosci 14:595-608, 2001

37. Sugawara T, Lewen A, Noshita N, Gasche Y, Chan PH: Effects of global ischemia duration on neuronal, astroglial, oligodendroglial, and microglial reactions in the vulnerable hippocampal CA1 subregion in rats. J Neurotrauma 19:85-98, 2002

38. Xu W, Mo J, Ocak U, Travis ZD, Enkhjargal B, Zhang T, Wu P, Peng J, Li T, Zuo Y, Shao A, Tang J, Zhang J, Zhang JH: Activation of melanocortin 1 receptor attenuates early brain injury in a rat model of subarachnoid hemorrhage viathe suppression of neuroinflammation through AMPK/TBK1/ NF-kappaB pathway in rats. Neurotherapeutics 2019 (Epub ahead of print)

39. Yu DK, Yoo KY, Shin BN, Kim IH, Park JH, Lee $\mathrm{CH}$, Choi $\mathrm{JH}$, Cho YJ, Kang IJ, Kim YM, Won MH: Neuronal damage in hippocampal subregions induced by various durations of transient cerebral ischemia in gerbils using Fluoro-Jade B histofluorescence. Brain Res 1437:50-57, 2012

40. Zeng X, Zhang S, Xu L, Yang H, He S: Activation of proteaseactivated receptor 2-mediated signaling by mast cell tryptase modulates cytokine production in primary cultured astrocytes. Mediators Inflamm 2013: 140812, 2013

41. Zhang S, Zeng X, Yang H, Hu G, He S: Mast cell tryptase induces microglia activation via protease-activated receptor 2 signaling. Cell Physiol Biochem 29:931-940, 2012

42. Zhang T, Xu S, Wu P, Zhou K, Wu L, Xie Z, Xu W, Luo X, Li P, Ocak U, Ocak PE, Travis ZD, Tang J, Shi H, Zhang JH: Mitoquinone attenuates blood-brain barrier disruption through Nrf2/PHB2/OPA1 pathway after subarachnoid hemorrhage in rats. Exp Neurol 317:1-9, 2019

43. Zhou Q, Wang YW, Ni PF, Chen YN, Dong HQ, Qian YN: Effect of tryptase on mouse brain microvascular endothelial cells via protease-activated receptor 2. J Neuroinflammation 15:248, 2018

44. Zuo G, Zhang T, Huang L, Araujo C, Peng J, Travis Z, Okada T, Ocak U, Zhang G, Tang J, Lu X, Zhang JH: Activation of TGR5 with INT-777 attenuates oxidative stress and neuronal apoptosis via cAMP/PKCepsilon/ALDH2 pathway after subarachnoid hemorrhage in rats. Free Radic Biol Med 143: 441-453, 2019

45. Zuo Y, Huang L, Enkhjargal B, Xu W, Umut O, Travis ZD, Zhang G, Tang J, Liu F, Zhang JH: Activation of retinoid X receptor by bexarotene attenuates neuroinflammation via PPAR gamma/ SIRT6/FoxO3a pathway after subarachnoid hemorrhage in rats. J Neuroinflammation 16:47, 2019 